\title{
BONA FIDE, STRONG-VARIABLE GALACTIC LUMINOUS BLUE VARIABLE STARS ARE FAST ROTATORS: DETECTION OF A HIGH ROTATIONAL VELOCITY IN HR CARINAE*
}

\author{
J. H. Groh ${ }^{1}$, A. Damineli ${ }^{2}$, D. J. Hillier ${ }^{3}$, R. Barbá ${ }^{4,5}$, E. Fernández-Lajús ${ }^{6}$, R. C. Gamen ${ }^{6}$, A. P. Moisés ${ }^{2}$, G. Solivella ${ }^{6}$, \\ AND M. TEODORO ${ }^{2}$ \\ ${ }^{1}$ Max-Planck-Institut für Radioastronomie, Auf dem Hügel 69, D-53121 Bonn, Germany; jgroh@mpifr-bonn.mpg.de \\ 2 Instituto de Astronomia, Geofísica e Ciências Atmosféricas, Universidade de São Paulo, Rua do Matão 1226, Cidade Universitária, 05508-090, \\ São Paulo, SP, Brazil \\ ${ }^{3}$ Department of Physics and Astronomy, University of Pittsburgh, 3941 O'Hara Street, Pittsburgh, PA 15260, USA \\ ${ }^{4}$ Departamento de Física, Universidad de La Serena, Benavente 980, La Serena, Chile \\ ${ }^{5}$ ICATE-CONICET, San Juan, Argentina \\ ${ }^{6}$ Facultad de Ciencias Astronómicas y Geofísicas, Universidad Nacional de La Plata, and Instituto de Astrofísica de La Plata (CCT La Plata-CONICET), \\ Paseo del Bosque S/N, B1900FWA, La Plata, Argentina \\ Received 2009 August 7; accepted 2009 September 18; published 2009 October 8
}

\begin{abstract}
We report optical observations of the luminous blue variable (LBV) HR Carinae which show that the star has reached a visual minimum phase in 2009. More importantly, we detected absorptions due to Si IV $\lambda \lambda 4088-4116$. To match their observed line profiles from 2009 May, a high rotational velocity of $v_{\text {rot }} \simeq 150 \pm 20 \mathrm{~km} \mathrm{~s}^{-1}$ is needed (assuming an inclination angle of $30^{\circ}$ ), implying that HR Car rotates at $\simeq 0.88 \pm 0.2$ of its critical velocity for breakup $\left(v_{\text {crit }}\right)$. Our results suggest that fast rotation is typical in all strong-variable, bona fide galactic LBVs, which present S-Dor-type variability. Strong-variable LBVs are located in a well-defined region of the HR diagram during visual minimum (the "LBV minimum instability strip"). We suggest this region corresponds to where $v_{\text {crit }}$ is reached. To the left of this strip, a forbidden zone with $v_{\text {rot }} / v_{\text {crit }}>1$ is present, explaining why no LBVs are detected in this zone. Since dormant/ex LBVs like P Cygni and HD 168625 have low $v_{\text {rot }}$, we propose that LBVs can be separated into two groups: fast-rotating, strong-variable stars showing S-Dor cycles (such as AG Car and HR Car) and slow-rotating stars with much less variability (such as P Cygni and HD 168625). We speculate that supernova (SN) progenitors which had S-Dor cycles before exploding (such as in SN 2001ig, SN 2003bg, and SN 2005gj) could have been fast rotators. We suggest that the potential difficulty of fast-rotating Galactic LBVs to lose angular momentum is additional evidence that such stars could explode during the LBV phase.
\end{abstract}

Key words: stars: atmospheres - stars: individual (HR Carinae) - stars: mass loss - stars: rotation - stars: variables: other - supergiants

\section{INTRODUCTION}

Massive stars are the main contributors to the input of ionizing photons, energy, and momentum into the interstellar medium and are responsible for a significant fraction of the chemical enrichment of their host galaxy. Evolutionary models predict the existence of a short-lived, transitional stage, usually referred to as the luminous blue variable (LBV) phase (Conti 1984; Humphreys \& Davidson 1994), characterized by a high massloss rate $\left(\dot{M} \sim 10^{-5}\right.$ to $\left.10^{-3} M_{\odot} \mathrm{yr}^{-1}\right)$. In the current picture of stellar evolution, LBVs are rapidly evolving massive stars in the transitory phase from being an O-type star burning hydrogen in its core to becoming a Wolf-Rayet (WR) type, helium coreburning star (Humphreys \& Davidson 1994; Maeder \& Meynet 2000b; Meynet \& Maeder 2000, 2003).

There is strong evidence that very massive stars lose the bulk of their mass through giant outbursts during the LBV phase (Smith \& Owocki 2006), similar to the one that occurred in Eta Carinae in the 1840s. Surprisingly, recent works suggest that some core-collapse supernovae (SNe) have LBV progenitors (e.g., Kotak \& Vink 2006; Smith et al. 2007, 2008a, 2008b; Gal-Yam et al. 2007; Trundle et al. 2008; Gal-Yam \& Leonard

\footnotetext{
* Based on observations made with the $1.6 \mathrm{~m}$ telescope at the Observatório Pico dos Dias (OPD-LNA, Brazil), with the $2.15 \mathrm{~m}$ telescope of the Complejo Astronomico El Leoncito (CASLEO, Argentina), and with the $2.2 \mathrm{~m}$ ESO telescope at La Silla (Chile) under program 083.D-0589. CASLEO is operated under agreement between CONICET, SECYT, and the National Universities of La Plata, Córdoba and San Juan, Argentina.
}

2009), which not only dramatically enhances the cosmological importance of LBVs but also poses a great challenge to the current paradigm of massive star evolution.

LBVs are rare: only a dozen or so are known in the Galaxy, and roughly only three of them, namely, AG Carinae, HR Carinae, and WRA 751, are confirmed strong-variable LBVs (Humphreys \& Davidson 1994; van Genderen 2001; Clark et al. 2005). These stars have been observed for a sufficiently long time to establish a record of strong photometric and spectroscopic variability which shows, undoubtedly, that both strong S-Dor-type variability and a circumstellar nebula are present. The spectroscopic and photometric behavior of these strong-variable LBVs are markedly different compared to other stars that are usually regarded as dormant LBVs, such as P Cygni and Eta Carinae (van Genderen 2001).

AG Car, HR Car, and WRA 751 are unstable and present cyclical strong S-Dor-type variability characterized by irregular visual magnitude changes on timescales of decades, with a typical amplitude of $\Delta V \simeq 1-2 \mathrm{mag}$, and corresponding changes in effective temperature and hydrostatic radius (van Genderen 2001). During visual minimum, the star is typically hot, while at visual maximum, a cooler effective temperature is obtained (e.g., van Genderen 1982). How the S-Dor-type variability relates to the powerful giant eruptions is not clear, although it could be possible that a relatively large amount of stellar mass, which is not ejected from the star, is taking part in the S-Dor-type variability (Groh et al. 2009, hereafter GHD09). 


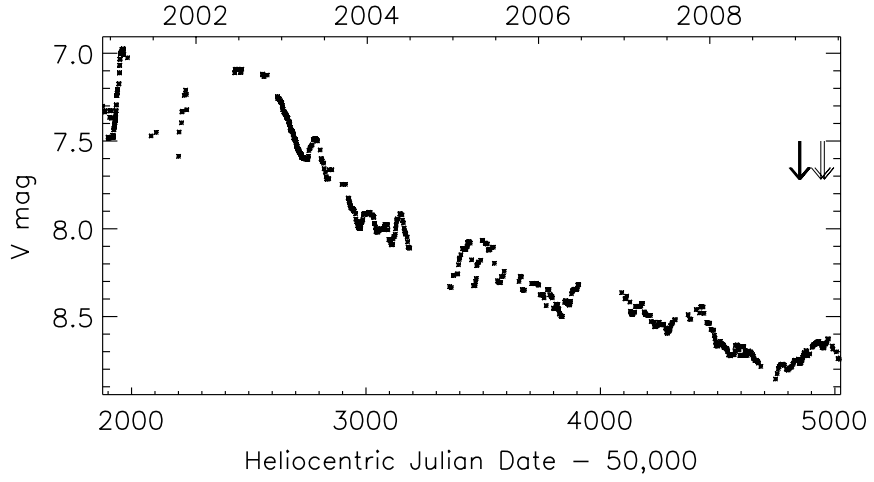

Figure 1. ASAS-3 CCD V-band light curve of HR Carinae from 2001 to 2009 The arrows indicate epochs when spectroscopic data were available during minimum.

This would suggest that the S-Dor-type variability is a failed giant eruption (GHD09).

At least for AG Car, two additional pieces of the puzzle are known: a significant reduction $(\sim 50 \%)$ in the inferred bolometric luminosity from visual minimum to maximum has been determined (GHD09) and a high rotational velocity has been obtained during minimum (Groh et al. 2006, hereafter GHD06). Whether the same is true for other bona fide LBVs, such as HR Car, still remains to be seen.

In this Letter, we report photometric and spectroscopic observations of the bona fide LBV HR Car obtained during 2009 (Section 2), showing that the star has entered a visual minimum phase of the S-Dor cycle. More importantly, we detected absorptions related to the high-excitation lines of Si IV $\lambda \lambda 4088-4116$ in HR Car. The results of our non-LTE spectroscopic modeling using the radiative transfer code CMFGEN are presented in Section 3. They suggest a photospheric nature for these lines, that HR Car has high rotational velocity $\left(v_{\text {rot }}\right)$, and that $v_{\text {rot }}$ is very high compared to the critical rotational velocity for breakup $\left(v_{\text {crit }}\right)$. In Section 4, we discuss the importance of such a detection for understanding the LBV phase and the subsequent evolution of very massive stars.

\section{OBSERVATIONS}

Optical CCD $V$-band photometry of HR Car was compiled from the ASAS-3 database (Pojmanski 2002) and averaged in bins of 15 days, with only high-quality (grade A) data being used. The ASAS-3 magnitudes were measured using a large aperture $\left(75^{\prime \prime}\right)$, but HR Car is by far the dominant source of light in the field. We estimate that the typical error of the 15-dayaveraged $V$-band magnitude is 0.03 mag. Figure 1 presents the $V$-band light curve of HR Car from 2001 to 2009, showing that a significant increase of $1.5 \mathrm{mag}$ occurred in this time span and that HR Car has entered a visual minimum phase of the S-Dor cycle.

Optical spectra of HR Car around the Si IV lines at $\lambda \lambda 4088$ 4116 were obtained using several facilities in the southern hemisphere. Medium resolution $(R \simeq 12,000)$ spectroscopic observations were made at the $1.6 \mathrm{~m}$ telescope of the Observatório Pico dos Dias (OPD-LNA, Brazil) and at the $2.15 \mathrm{~m}$ telescope of the Complejo Astronomico El Leoncito (CASLEO, Argentina). High-resolution spectra ( $R \simeq 48,000$ ) were gathered using the Fiber-fed Extended Range Optical Spectrograph (FEROS; Kaufer et al. 1999) mounted on the $2.2 \mathrm{~m}$ telescope of the Max-Planck-Gesellschaft/European Southern Observatory (MPG/ESO, Chile). The reduction of the spectroscopic data,

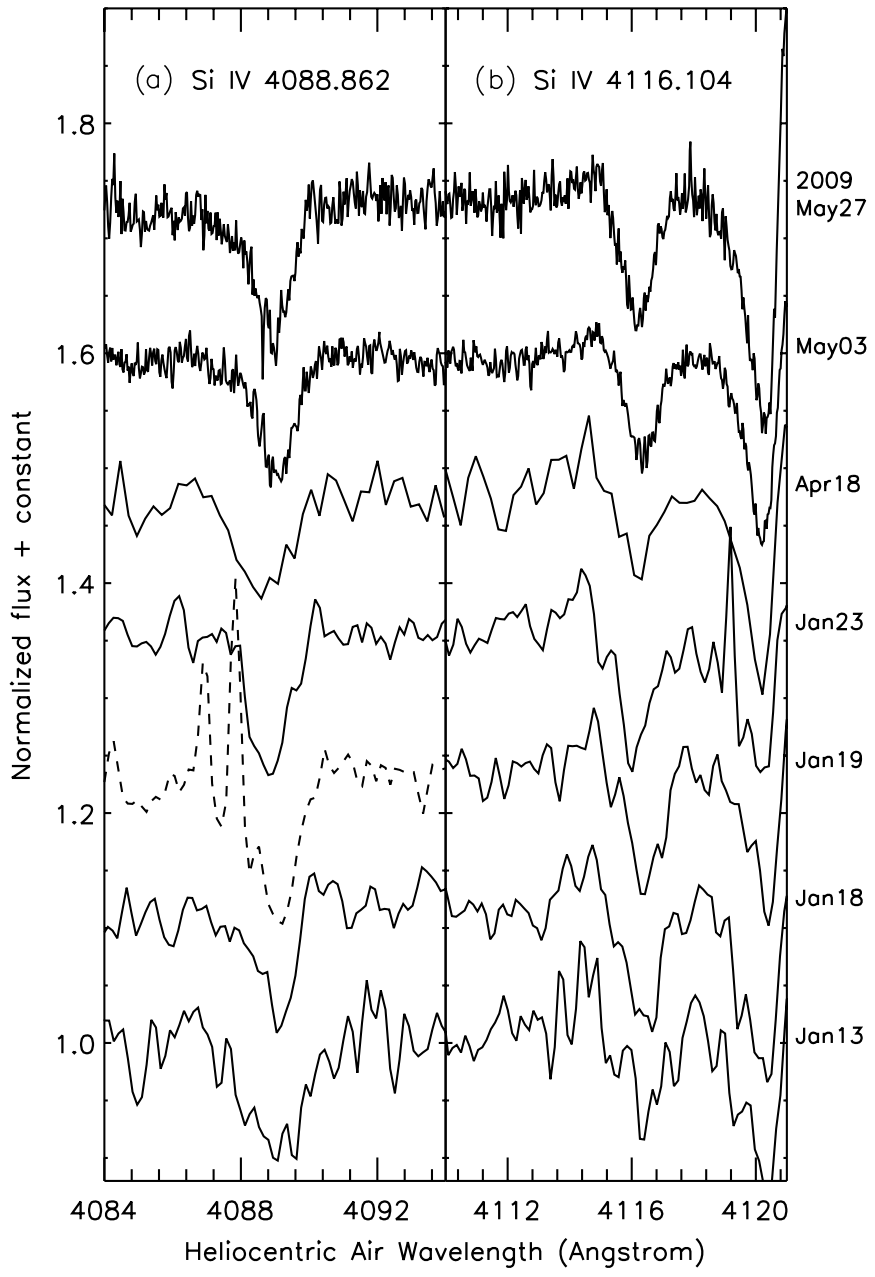

Figure 2. Optical spectra of HR Car around Si IV $\lambda \lambda 4088-4116$. From bottom to top, we show spectra obtained from 2009 January 13 until 2009 May 27. The blue wing of Si IV $\lambda 4088$ was hit by cosmic rays on 2009 January 18 and is shown by the dashed line.

including bias and sky subtraction, flat fielding, spectrum extraction, wavelength calibration, correction to the heliocentric frame, and continuum normalization, was carried out using standard IRAF ${ }^{7}$ routines. A systemic velocity of $-10 \mathrm{~km} \mathrm{~s}^{-1}$ (Weis et al. 1997) was subtracted from the observed spectra. Table 1 summarizes the observational data of HR Car available during visual minimum.

Figure 2 presents a montage of the observed Si IV $\lambda \lambda 4088$ 4116 line profiles of HR Car during our 2009 monitoring. The use of different instruments over five months confirms that the detection is not transient and, undoubtedly, absorptions due to Si IV $\lambda \lambda 4088-4116$ lines are typical in HR Car during visual minimum.

\section{MODELING AND RESULTS}

In the case of the prototypical LBV AG Car, the relatively broad absorptions due to Si IV $\lambda \lambda 4088-4116$ are caused by the extremely fast rotation, $v_{\text {rot }} / v_{\text {crit }} \gtrsim 0.86$ (GHD06). Here, we also use these Si IV lines to derive the rotational velocity of HR Car, performing a detailed spectroscopic analysis similar to GHD06, using the radiative transfer code CMFGEN (Hillier \&

\footnotetext{
7 IRAF is distributed by the National Optical Astronomy Observatory, which is operated by the Association of Universities for Research in Astronomy, Inc., under cooperative agreement with the National Science Foundation.
} 
Table 1

Journal of Optical Spectroscopy of HR Car during the 2009 Visual Minimum

\begin{tabular}{lcccr}
\hline \hline \multicolumn{1}{c}{ Date } & $V$ & Telescope/Instrument & Spectral Range $(\AA)$ & $R$ \\
\hline 2009 Jan 13 & 8.74 & 2.15 m CASLEO/REOSC & $3650-6130$ & 12000 \\
2009 Jan 18-19-23 & 8.77 & 2.15 m CASLEO/REOSC & $3650-6130$ & 12000 \\
2009 Apr 18 & 8.66 & 1.6 m OPD-LNA/Coude & $3880-4390$ & 12000 \\
2009 May 3-27 & 8.67 & 2.2 m MPG-ESO/FEROS & $3600-9200$ & 48000 \\
\hline
\end{tabular}

Miller 1998) and the two-dimensional (2D) code of Busche \& Hillier (2005, hereafter BH05).

CMFGEN solves the statistical equilibrium equations simultaneously to the radiative transfer in the co-moving frame of the outflow, in spherical symmetry, and assuming steady state. The code computes line and continuum formation in the non-LTE regime, and each model is specified by the stellar luminosity $L_{\star}$ and effective temperature $T_{\text {eff }},{ }^{8}$ the wind mass-loss rate $\dot{M}$, volume filling factor $f$, and terminal velocity $v_{\infty}$, and the chemical abundances $Z_{i}$ of the included species. Since the momentum equation of the wind is not solved, a beta-type velocity law is adopted and modified at depth to smoothly match a hydrostatic structure at $R_{\star}$. The hydrostatic equation is iterated in order to produce a quasi-hydrostatic structure which extends inward until the inner boundary of the model (at a Rosseland optical depth of 100). Line blanketing is fully taken into account, and our models include tens of thousands of spectral lines in non-LTE. The atomic model used for HR Car is similar to the one used for AG Car (GHD09) and included lines of H, He, C, N, O, Na, $\mathrm{Mg}, \mathrm{Al}, \mathrm{Si}, \mathrm{Cr}, \mathrm{Mn}, \mathrm{Fe}, \mathrm{Co}$, and $\mathrm{Ni}$.

We assumed a depth-independent turbulent velocity of $20 \mathrm{~km} \mathrm{~s}^{-1}$ in the computation of the observed spectrum, which is an upper limit, since a higher turbulent velocity would yield a detectable redshift, which is not observed, of the emission lines with high opacity, such as the stronger lines of $\mathrm{H}$ I and He I. Therefore, microturbulence cannot explain the broadening seen in the high-ionization Si IV $\lambda \lambda$ 4088-4116 lines, and we suggest these lines to be rotationally broadened (note that other broadening mechanisms, such as convection or other kind of surface activity, have yet to be detected in LBVs and, thus, are not expected to be present in AG Car, although our data do not rule them out).

The effects of rotation on the line profiles were investigated using the BH05 code, which allows the computation of the spectrum in 2D geometry for a given inclination angle $i$. No equatorial or polar density enhancements were taken into account, since our goal was to investigate the broadening of the photospheric lines. Therefore, we computed a grid of synthetic line profiles with a stellar rotational velocity ranging from 0 to $200 \mathrm{~km} \mathrm{~s}^{-1}$ in increments of $5 \mathrm{~km} \mathrm{~s}^{-1}$, and a best fit was obtained by visual inspection. We assume that the stellar rotational axis of HR Car is aligned with the polar axis of its bipolar nebula, which is inclined at $i=30^{\circ} \pm 10^{\circ}$ (Nota et al. 1997).

In a dense stellar wind, ionization stratification occurs, and lines from ions of different ionization potentials will form at different distances $r$ from the star. The Si IV $\lambda \lambda 4088-4116$ lines are the highest ionization lines present in the optical spectrum of HR Car and, therefore, should be formed closest to the stellar photosphere. Since the azimuthal velocity component is proportional to $r^{-1}, \mathrm{Si}$ IV $\lambda \lambda 4088-4116$ are ideal for deriving

\footnotetext{
8 The effective temperature is defined as the temperature where the Rosseland optical depth is $2 / 3$, i.e., $T_{\text {eff }}=T\left(\tau_{\text {Ross }}=2 / 3\right)$.
}

the rotational velocity of HR Car. Our modeling shows that other optical lines which are occasionally seen in absorption in LBV spectra, such as N II $\lambda \lambda 4601-4607-4630-4643$ and Si III $\lambda \lambda 4553-4558-4575$, are actually formed in the wind of HR Car, far from the stellar photosphere. As a consequence, their broadening is dominated by the wind velocity field (see discussion in BH05 and GHD06).

Figure 3 compares the absorption profiles of Si IV $\lambda \lambda 4088$ 4116 observed in 2009 May 3 (which has the highest signalto-noise ratio and spectral resolution of our sample) with our best CMFGEN model, which has the following parameters: $L_{\star}=5.0 \times 10^{5} L_{\odot}\left(\right.$ assuming $\mathrm{A}_{V}=3.1$ and $d=5 \mathrm{kpc}$; van Genderen et al. 1991; Hutsemekers \& van Drom 1991), $R_{\star}=70 R_{\odot}, R_{\text {phot }}=75 R_{\odot}, T_{\text {eff }}=17,900 \mathrm{~K}, \mathrm{He} / \mathrm{H}=0.4$ (by number), $v_{\infty}=120 \mathrm{~km} \mathrm{~s}^{-1}, \beta=1, \dot{M}=7 \times 10^{-6} M_{\odot} \mathrm{yr}^{-1}$, and $f=0.25$. In a forthcoming full paper, we will present the results of the detailed spectroscopic analysis of HR Car during minimum, including diagnostics for each of the parameters assumed above. Nevertheless, for reasonable changes in the above parameters, the derived rotational velocity of HR Car changes only slightly and the conclusions of this Letter remain valid.

Similar to AG Car (GHD06), the observed absorption profiles of both Si IV lines are significantly broader and shallower than the non-rotating model. Our model predicts that the Si IV lines are formed very close to the stellar photosphere, where the wind is still accelerating and, therefore, the line broadening caused by the wind velocity field is negligible. Consequently, most of the line broadening should be due to rotation. According to our rotating models computed with the $\mathrm{BH} 05$ code, a rotational velocity of $v_{\text {rot }} \simeq 150 \pm 20 \mathrm{~km} \mathrm{~s}^{-1}$ is required to reproduce the observed shape of the Si IV $\lambda \lambda 4088-4116$ lines in HR Car (Figure 3).

How does the rotational velocity of HR Car compare to its critical velocity for breakup? An estimation of $v_{\text {crit }}$ can be calculated following Maeder \& Meynet (2000a) once the stellar mass $M, L_{\star}, R_{\star}$, and the Eddington parameter $\Gamma$ are known. The critical parameter for obtaining a precise value of $v_{\text {rot }} / v_{\text {crit }}$ is $M$, which can be estimated based on the evolutionary models from Meynet \& Maeder (2003). Such models predict that, based on our derived value of $L_{\star}=5.0 \times 10^{5} L_{\odot}$, the initial mass of HR Car should have been roughly $M_{\text {ini }} \simeq 50 \pm 10 M_{\odot}$. While there are no evolutionary models available in the literature specifically for $M_{\text {ini }}=50 M_{\odot}$, we estimate that stars such as HR Car will reach the LBV phase with $M \sim 25 M_{\odot}$, since evolutionary models predict that a $40 M_{\odot}$ star reaches the LBV phase with $20 M_{\odot}$, while a $60 M_{\odot}$ star reaches the LBV phase with $30 M_{\odot}$ (Meynet \& Maeder 2003).

Assuming an admittedly uncertain $M=25 M_{\odot}$, our CMFGEN model of HR Car predicts $\Gamma \simeq 0.80$, and we derive $v_{\text {crit }} \simeq 170 \pm 20 \mathrm{~km} \mathrm{~s}^{-1}$ and $v_{\text {rot }} / v_{\text {crit }} \simeq 0.88 \pm 0.2$. Therefore, similar to AG Car (GHD06), HR Car is also likely rotating very close to, if not at, its critical velocity for breakup. 


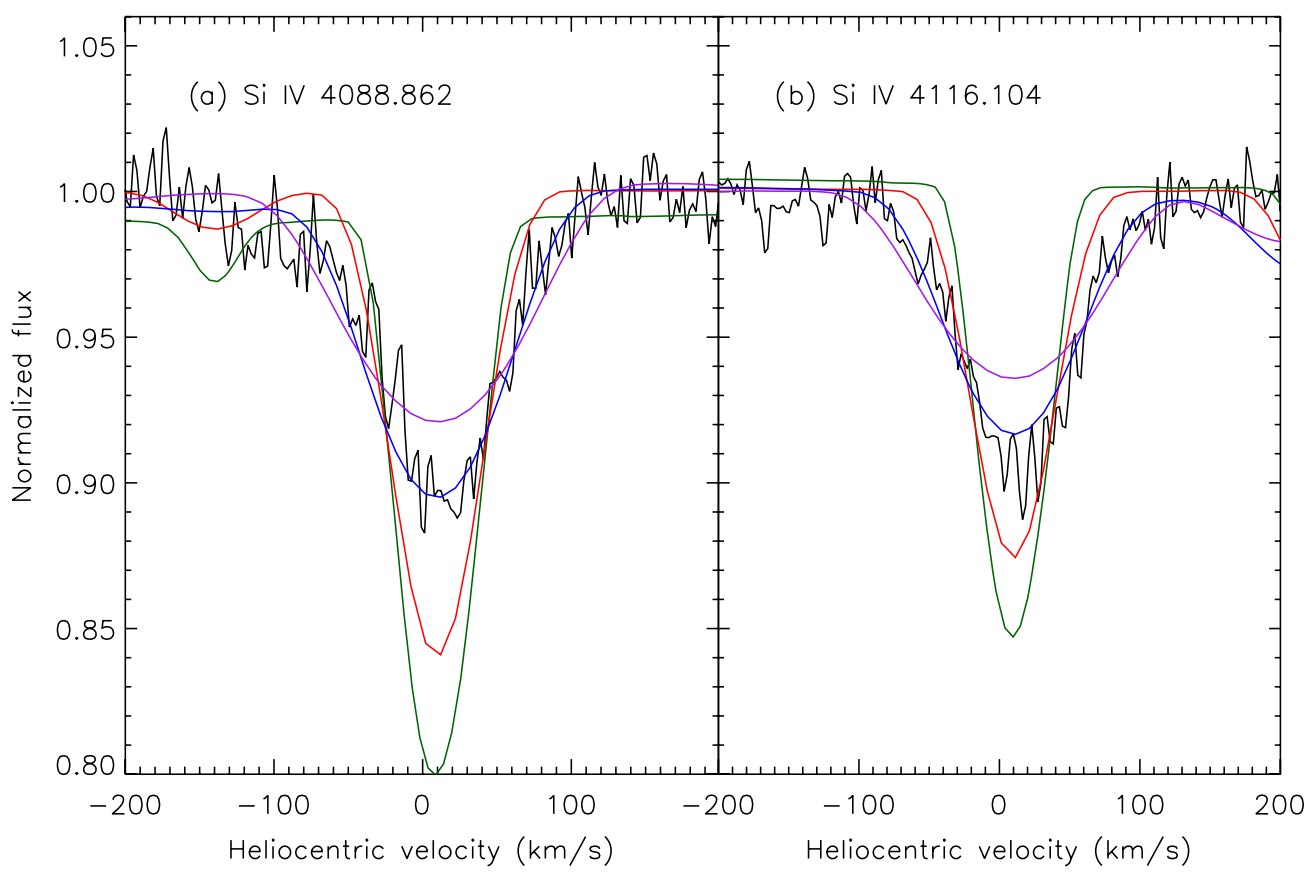

Figure 3. Comparison between the Si IV $\lambda \lambda 4088-4116$ line profiles of HR Car observed on 2009 May 3 with synthetic model spectra computed with the 2D code from BH05 for different rotational velocities, assuming $i=30^{\circ}: 0 \mathrm{~km} \mathrm{~s}^{-1}$ (green), $75 \mathrm{~km} \mathrm{~s}^{-1}$ (red), $150 \mathrm{~km} \mathrm{~s}^{-1}$ (blue), and $200 \mathrm{~km} \mathrm{~s}^{-1}$ (purple).

\section{DISCUSSION: STRONG-ACTIVE, BONA FIDE GALACTIC LBVs ARE FAST ROTATORS}

Several theoretical works have long predicted that LBVs should have high rotational velocities (e.g., Humphreys \& Davidson 1994; Langer et al. 1999; Maeder \& Meynet 2000a; Maeder \& Desjacques 2001; Dwarkadas \& Owocki 2002; Aerts et al. 2004), but direct observational detection through the broadening of spectral lines has been elusive until recently, in particular because high-resolution observations of LBVs in the blue part of the optical spectrum are scarcely available during visual minimum. The detection of high rotational velocity in two of the most variable LBVs in the Galaxy, AG Car (GHD06) and now HR Car (Section 3), raises the obvious question: are all LBVs fast rotators?

The presence of fast rotation in two out of three ${ }^{9}$ of the galactic bona fide LBVs that have a circumstellar nebula and strong S-Dor-type variability is probably not a coincidence, and we suggest that fast rotation is typical in such stars. Perhaps, fast rotation might even be a key ingredient for the instability that causes the S-Dor cycles in LBVs. If that is true, we should expect that fast rotation is also present during visual minimum in extragalactic LBVs, such as R127 and S Doradus in the LMC. An obvious test case for such a hypothesis would be R127, which has been undergoing a visual minimum phase since 2007 (Walborn et al. 2008).

Figure 4 presents the position of AG Car and HR Car in the HR diagram based on their updated stellar parameters obtained in GHD09 and in this work. Previous works have recognized that, during visual minimum, LBVs are located in a well-defined region of the HR diagram, the so-called LBV minimum instability strip (Wolf 1989; Clark et al. 2005). Our results suggest that the LBV minimum instability strip is considerably steeper than previously determined, being characterized by $\log \left(L / L_{\odot}\right)=4.54 \log \left(T_{\text {eff }} / \mathrm{K}\right)-13.61$ (red dashed

\footnotetext{
9 Unfortunately, no high-resolution observations of WRA 751 during minimum are available to us to check if it is a fast rotator.
}

line in Figure 4). More importantly, we suggest that the LBV minimum instability strip corresponds to the region where critical rotation is reached for LBVs with strong S-Dor-type variability. When LBVs are evolving toward maximum, the star moves far from the LBV minimum instability strip (to the right in the HR diagram), and $v_{\text {rot }} / v_{\text {crit }}$ decreases considerably (GHD06). The region in the HR diagram on the left side of the LBV minimum instability strip would be populated by unstable LBV stars with $v_{\text {rot }} / v_{\text {crit }}>1$, which would make it a "forbidden region" for LBVs. Indeed, no confirmed strong-variable LBV is seen in this region (van Genderen 2001; Clark et al. 2005).

A corollary of the relationship between S-Dor-type instability and fast rotation that we suggest in this Letter regards $\mathrm{SNe}$ which likely had an LBV progenitor. Some of these LBVs showed evidence for S-Dor-type variability before exploding as a $\mathrm{SN}$, such as the progenitors of SN 2001ig, SN 2003bg (Kotak \& Vink 2006), and SN 2005gj (Trundle et al. 2008). It could be very likely that these massive stars were fast rotators before exploding.

What about dormant LBVs that have not presented evidence for strong S-Dor-type variability for a century or more? From these, only Eta Car has shown indirect evidence of fast rotation, through the presence of a latitude-dependent wind (Smith et al. 2003; Weigelt et al. 2007). Interestingly, at least two other confirmed LBVs that do not show S-Dor-type variability appear to rotate slowly. $\mathrm{P}$ Cygni has evidence of a relatively low rotational velocity of $50-60 \mathrm{~km} \mathrm{~s}^{-1}$ (Najarro et al. 1997), ${ }^{10}$ a property also shared by HD 168625 , for which we measured $v \sin i \simeq 40 \mathrm{~km} \mathrm{~s}^{-1}$ based on optical photospheric $\mathrm{N}$ II lines and a similar methodology as in the present work (J. H. Groh 2009 , in preparation). Assuming $i \simeq 60^{\circ}$ (Smith 2007), we obtain $v_{\text {rot }} \simeq 45 \mathrm{~km} \mathrm{~s}^{-1}$. For both P Cygni and HD 168625 , the critical velocity for breakup should be on the order of

\footnotetext{
10 Assuming the rotational axis of P Cygni is aligned with part of its nebula, which is seen at $i \sim 45^{\circ}$ (Smith \& Hartigan 2006). Note that this assumption is not crucial for the results of this Letter.
} 


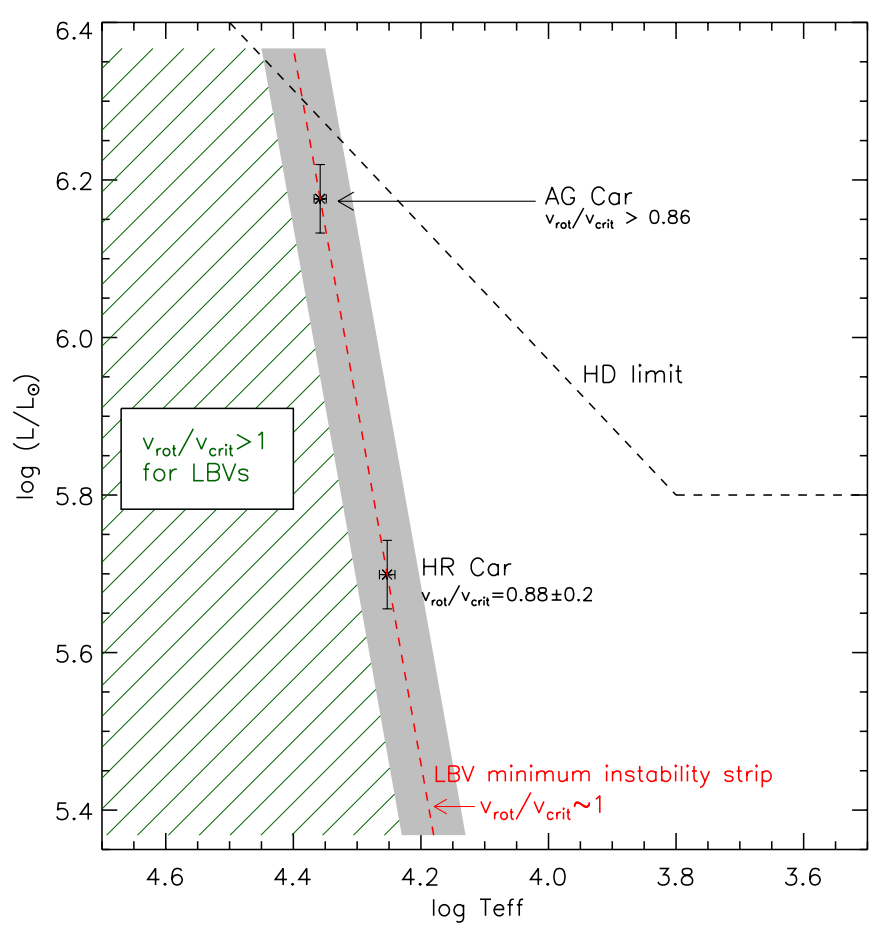

Figure 4. HR diagram showing the position of the strong-variable LBVs AG Car and HR Car during visual minimum, according to the updated stellar parameters determined in GHD09 and in this work. A revised position for the LBV minimum instability strip is provided (red dashed line), and is considerably steeper than previous determinations (Wolf 1989; van Genderen 2001; Clark et al. 2005). Notice that AG Car and HR Car are both fast rotators during minimum, which suggests that the LBV minimum instability strip corresponds to the location where critical rotation is reached for strong-variable LBVs. The location of the Humphreys-Davidson limit (Humphreys \& Davidson 1994) is shown (black dashed line).

$150-200 \mathrm{~km} \mathrm{~s}^{-1}$, meaning that $v_{\text {rot }} / v_{\text {crit }}$ should be $\sim 0.2-0.3$ for these stars.

We suggest that LBVs, at least in our Galaxy, can be separated into two groups: group I, composed of fast-rotating, strongvariable LBVs such as AG Car and HR Car, and group II, composed of slow-rotating, weak variable LBVs such as P Cygni and HD 168625. While an LBV can in principle migrate from one subgroup to the other, for instance, after a giant eruption, these LBV groups might also follow different evolutionary paths during and after the LBV phase, possibly yielding significantly different SN progenitors.

In particular, fast-rotating $\mathrm{LBVs}$ might struggle to lose enough angular momentum through winds and giant eruptions. Fast-rotating stars are supposed to have a latitude-dependent, polar-enhanced wind (Owocki et al. 1996) which, depending on the mass-loss rate, will not remove as much angular momentum as in the case of a spherical wind (see discussion in Maeder 2002). In addition, LBVs might not lose enough angular momentum during giant outbursts, similar to the one that occurred in Eta Car in the 1840s, since almost $75 \%$ of the mass was lost at latitudes higher than $45^{\circ}$ (Smith 2006). Although current evolutionary models predict that fast-rotating LBVs should spin down (Meynet \& Maeder 2003), the models do not include angular momentum and mass losses through a giant outburst, which is a key factor during the LBV phase (Smith \& Owocki 2006). In the Galaxy, there are at least three examples of stars which had a giant eruption, lost significant amounts of mass (several $M_{\odot}$ ), but are still currently fast rotators: Eta Car
(Smith et al. 2003; Weigelt et al. 2007), AG Car (Groh et al. 2006), and HR Car (this work).

As a consequence, if fast-rotating LBVs are to evolve to WR stars without losing enough angular momentum, they would produce a population of significantly fast-rotating WR stars, which is yet unseen in the Galaxy ${ }^{11}$ and not predicted by evolutionary models (Meynet \& Maeder 2003). An alternative evolutionary path would be that LBVs could fail to lose an additional significant amount of mass and thus, instead of becoming a WR star, would dramatically explode as a corecollapse SN anytime during the LBV phase (e.g., Smith et al. 2007; Smith 2008). We suggest that the potential difficulty of fast-rotating Galactic LBVs to lose angular momentum is an additional evidence that LBVs such as AG Car and HR Car could explode during the LBV phase.

We thank Nathan Smith, Gerd Weigelt, Thomas Driebe, and an anonymous referee for invaluable comments. J.H.G. thanks the Max Planck Society for financial support. A.D., A.M., and M.T. acknowledge support from FAPESP and CNPq, D.J.H. from NSF grant AST-0507328, and R.H.B. partially from Universidad de La Serena (project DIULS CD08102).

Facilities: OPD/LNA, CASLEO, ESO

\section{REFERENCES}

Aerts, C., Lamers, H. J. G. L. M., \& Molenberghs, G. 2004, A\&A, 418, 639 Annuk, K. 1990, Acta Astron., 40, 267

Busche, J. R., \& Hillier, D. J. 2005, AJ, 129, 454

Clark, J. S., Larionov, V. M., \& Arkharov, A. 2005, A\&A, 435, 239

Conti, P. S. 1984, in IAU Symp. 105, Observational Tests of the Stellar Evolution

Theory, ed. A. Maeder \& A. Renzini (Dordrecht: Kluwer), 233

Dwarkadas, V. V., \& Owocki, S. P. 2002, ApJ, 581, 1337

Gal-Yam, A., \& Leonard, D. C. 2009, Nature, 458, 865

Gal-Yam, A., et al. 2007, ApJ, 656, 372

Groh, J. H., Hillier, D. J., \& Damineli, A. 2006, ApJ, 638, L33

Groh, J. H., et al. 2009, ApJ, 698, 1698

Hillier, D. J., \& Miller, D. L. 1998, ApJ, 496, 407

Humphreys, R. M., \& Davidson, K. 1994, PASP, 106, 1025

Hutsemekers, D., \& van Drom, E. 1991, A\&A, 248, 141

Kaufer, A., et al. 1999, Messenger, 95, 8

Kotak, R., \& Vink, J. S. 2006, A\&A, 460, L5

Langer, N., García-Segura, G., \& Mac Low, M.-M. 1999, ApJ, 520, L49

Maeder, A. 2002, A\&A, 392, 575

Maeder, A., \& Desjacques, V. 2001, A\&A, 372, L9

Maeder, A., \& Meynet, G. 2000a, A\&A, 361, 159

Maeder, A., \& Meynet, G. 2000b, ARA\&A, 38, 143

Massey, P. 1980, ApJ, 236, 526

Meynet, G., \& Maeder, A. 2000, A\&A, 361, 101

Meynet, G., \& Maeder, A. 2003, A\&A, 404, 975

Najarro, F., Hillier, D. J., \& Stahl, O. 1997, A\&A, 326, 1117

Nota, A., Smith, L., Pasquali, A., Clampin, M., \& Stroud, M. 1997, ApJ, 486, 338

Owocki, S. P., Cranmer, S. R., \& Gayley, K. G. 1996, ApJ, 472, L115

Pojmanski, G. 2002, Acta Astron., 52, 397

Smith, N. 2006, ApJ, 644, 1151

Smith, N. 2007, AJ, 133, 1034

Smith, N. 2008, in ASP Conf. Ser. 388, Mass Loss from Stars and the Evolution of Stellar Clusters, ed. A. de Koter, L. J. Smith, \& L. B. F. M. Waters (San Francisco, CA: ASP), 129

Smith, N., Davidson, K., Gull, T. R., Ishibashi, K., \& Hillier, D. J. 2003, ApJ, 586,432

Smith, N., \& Hartigan, P. 2006, ApJ, 638, 1045

11 Massey (1980) suggested a high rotational velocity of $\sim 500 \mathrm{~km} \mathrm{~s}^{-1}$ for the WN5 star HD 193077, based on broad absorptions due to high Balmer and He I lines. However, Annuk (1990) proposed that HD193077 is a WR+O binary and that the broad absorption lines are formed in the $\mathrm{O}$ star. Even if the absorption lines were formed in the WR wind, they should be formed sufficiently far from the hydrostatic core that the broadening would be dominated by the wind acceleration. 
Smith, N., \& Owocki, S. P. 2006, ApJ, 645, L45

Smith, N., et al. 2007, ApJ, 666, 1116

Smith, N., et al. 2008a, ApJ, 686, 467

Smith, N., et al. 2008b, ApJ, 686, 485

Trundle, C., Kotak, R., Vink, J. S., \& Meikle, W. P. S. 2008, A\&A, 483, L47

van Genderen, A. M. 1982, A\&A, 112, 61

van Genderen, A. M. 2001, A\&A, 366, 508 van Genderen, A. M., Robijn, F. H. A., van Esch, B. P. M., \& Lamers, H. J. G. 1991, A\&A, 246, 407

Walborn, N. R., et al. 2008, ApJ, 683, L33

Weigelt, G., et al. 2007, A\&A, 464, 87

Weis, K., Duschl, W. J., Bomans, D. J., Chu, Y.-H., \& Joner, M. D. 1997, A\&A, 320,568

Wolf, B. 1989, A\&A, 217, 87 